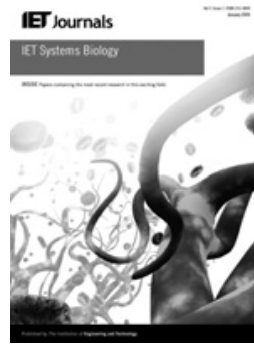

ISSN $1751-8849$

\title{
Critical perspective on the consequences of the limited availability of kinetic data in metabolic dynamic modelling
}

\author{
R.S. Costa D. Machado I. Rocha E.C. Ferreira \\ IBB-Institute for Biotechnology and Bioengineering, Centre of Biological Engineering, University of Minho, \\ Campus de Gualtar, P-4710-057 Braga, Portugal \\ E-mail: rafacosta@deb.uminho.pt
}

\begin{abstract}
Detailed kinetic models at the network reaction level are usually constructed using enzymatic mechanistic rate equations and the associated kinetic parameters. However, during the cellular life cycle thousands of different reactions occur, which makes it very difficult to build a detailed large-scale ldnetic model. In this work, we provide a critical overview of specific limitations found during the reconstruction of the central carbon metabolism dynamic model from $E$. coli (based on kinetic data available). In addition, we provide clues that will hopefully allow the systems biology community to more accurately construct metabolic dynamic models in the future. The difficulties faced during the construction of dynamic models are due not only to the lack of kinetic information but also to the fact that some data are still not curated. We hope that in the future, with the standardization of the in vitro enzyme protocols the approximation of in vitro conditions to the in vivo ones, it will be possible to integrate the available kinetic data into a complete large scale model. We also expect that collaborative projects between modellers and biologists will provide valuable kinetic data and permit the exchange of important information to solve most of these issues.
\end{abstract}

\section{Background}

During the last years, the tremendous increase in the availability of biological data because of novel highthroughput analytical techniques, such as the ones used in metabolomics, proteomics and transcriptomics, allowed an unprecedented insight on intracellular dynamics [1, 2]. However, more detailed mathematical models are necessary to integrate these experimental data in the interest of understanding metabolism under a quantitative aspect [3].

A variety of macroscopic mathematical models can be found in the literature for the growth of microorganisms with successful industrial applications [4-6]. However, when a more detailed investigation of the complexity of metabolic and/or signalling pathways is desired, models that incorporate intracellular phenomena are necessary. The most suitable large-scale cell models are stoichiometric models and models that account for dynamics at the enzyme level [7-9]. In many cases, a well-constructed dynamic model is preferable because it provides a basis for testing hypotheses under different perturbations over time and other analyses, such as metabolic control analysis [10].

Dynamic modelling of biochemical networks, although not a recent topic, has evolved substantially in recent times, aided by the arrival of completely sequenced genomes [11], the development of technologies to rapidly obtain quantitative measurements for multiple metabolites $[12,13]$ and the completion of publicly available metabolic databases [14-20].

Non-linear ordinary differential equations (ODE's) systems are the most commonly applied techniques in this context

$$
\frac{\mathrm{d} C_{i}}{\mathrm{~d} t}=\sum_{j=1}^{r} N_{i j} v_{j}-\mu C_{i}
$$

where $C_{i}$ stands for the intracellular concentration of metabolite $i$, and $N_{i j}$ is the stoichiometric coefficient of metabolite $i$ in reaction $j$. The term $v_{j}$ is the rate of reaction $j$, which depends non-linearly on the metabolites concentrations and kinetic parameters, $r$ is the number of reactions in the network and $\mu$ is the specific growth rate.

These models require prior knowledge on the network structure and detailed kinetic rate laws. They also depend on a large number of enzymatic kinetic parameter values and a description of the experimental conditions under which they were determined. However, one of the major problems of setting up large-scale dynamic mechanistic models is precisely the lack of kinetic information. In addition, usually obtained experimental results are not directly comparable if the assay conditions are not standardised. Despite recent achievements in enzymatic high-throughput assays, the kinetic parameters are usually unknown for a large number of enzymes or species, or are 
available in the literature/databases only as general values obtained by in vitro experiments by enzymologists $[15,16]$. These kinetic parameters should be used with care by modellers, since enzymologists in general work under optimal conditions for the enzyme and do not perform the enzyme characterisation under physiological conditions, restricting the in silico applicability of these parameters [21]. Consequently, for the integration of kinetic information from many different labs under different conditions, it is that the uniformity of the experimental procedures should also be representative of the in vivo conditions.

On the other hand, the developments in modern highthroughput methods have allowed the generation of in vivo time series or steady-state data of metabolite concentrations and metabolic fluxes, and enable us to tackle also parameter estimation by collective fitting $[8,22,23]$. The clear advantage of using a reverse approach is that the information from modern high-throughput techniques is collected within the same organism and under the same experimental conditions. Although these high-throughput data are becoming increasingly available, challenges in kinetic parameter estimation and model structure identification remain vast. For example, the difficulty of extracting precise kinetic parameters from collective fitting can reduce the extrapolation ability of the dynamic model [24].

The aim of this work was to provide a critical overview of specific limitations found during the reconstruction of dynamic metabolic models. Emphasis is given on providing clues that will hopefully contribute to the standardisation of experimental procedures and efforts to bring the systems biology community and biologists closer. Furthermore, alternative methods to overcome some of the limitations on kinetic information are described.

\section{Use of in vitro kinetic parameters in metabolic models}

The applicability of parameter values measured in vitro to a particular in vivo system has been the subject of much debate in the last years. Wright et al. [25] and Bakker et al. [26] reported two of the first works that describe in vivo systems on the basis of in vitro kinetics. In addition, the dynamic model constructed by Teusink et al. [21] intended to test whether in vivo systems could be described on the basis of the kinetic parameters determined from isolated enzymes. Besides illustrating some of the difficulties involved in the construction of large-scale models based on in vitro kinetics data, these authors also found that in many cases there was sufficient consistency of metabolite concentrations and kinetic parameters. More recently, Ishii et al. [27] estimated experimentally unknown parameter values of three isolated enzymes (Glk, Glucokinase; Pgi, phosphoglucoisomerase and PfkA, phosphofructokinase) from Escherichia coli under the same experimental conditions. These kinetic parameters were then incorporated in a model and the simulations compared with time course metabolite concentrations. The successful application of this approach indicates that when automated enzyme assays and accurate in vitro kinetic parameters are provided, a living cell model can be built.

Ideally, all metabolite and enzyme data should come from the same organism, tissue, extract and also from the same experimental conditions (e.g. $\mathrm{pH}$, temperature, redox balance, ionic strength etc.). Unfortunately, these data are often measured under non-standard conditions and furthermore published data often lack some of this crucial information for the set up of dynamic models. In other words, an environment that fails to mimic an in vivo system to determine the kinetic parameters may contribute to flawed model predictions. In addition, often all the necessary kinetic information for model construction cannot be taken from a single data source. Therefore their use is limited and it is difficult to set up large-scale kinetic dynamic models.

Thus, the applicability of the dynamic modelling has been limited to biochemical networks of limited size. An example of a comparison between dynamic simulation results from a small biochemical network model and experimental time series data for $E$. coli is shown in Fig. 1. The model was constructed based only on kinetic information from the literature and public web databases, obtained in conditions similar to the experimental data. The simulation results, when compared with the measured time series data for metabolite concentrations after addition of glucose, show a reasonable agreement (Fig. 1).

In the course of the reconstruction of the dynamic model for the central carbon metabolism of $E$. coli we have encountered several problems with the available enzyme kinetic data. These problems, together with some suggestions on how to integrate useful information from available databases, will be described in the next section.

\section{Limitations of published kinetic data - some considerations to set up dynamic models}

One of the steps of a typical dynamic model building cycle is the collection of various kinetic/thermodynamic parameter values with the respective kinetic rate laws from databases or the literature and/or their estimation from time course experimental data. However, while pathways information has been compiled in several publicly available databases and biochemistry textbooks $[17,30]$, there are currently few databases collecting kinetic data (see Table 1). In addition, the

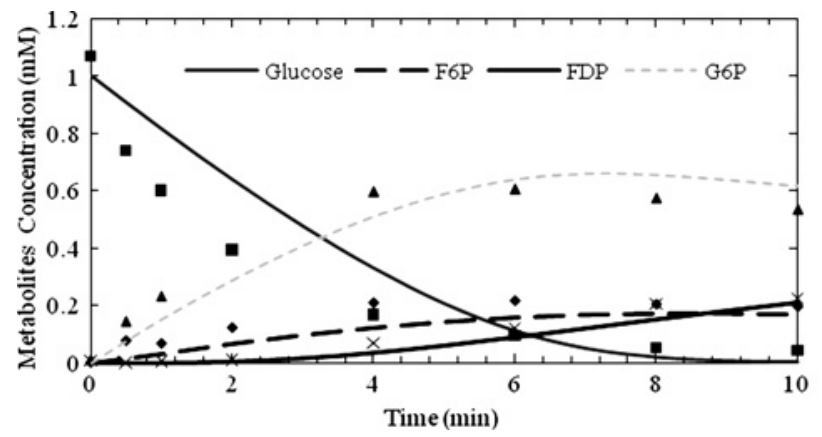

Fig. 1 Comparison between simulated (lines) and experimental data (symbols) for Glucose (ם); fructose-6-phosphate, F6P ( ); fructose 1,6-diphosphate, FDP $(\times)$ and glucose-6-phosphate, G6P ( $\mathbf{\Delta})$

Lines represent the simulation using rate laws for each reaction based on Ishil et al. [27]. Kinetic parameters $\quad\left(V_{\max , \mathrm{GLK}}=158 \mathrm{U} / \mathrm{mg}\right.$, $K_{\mathrm{GLK}, \text { glucose }}=0.15 \mathrm{mM}, \quad K_{\mathrm{GLK}, \text { atp }}=0.5 \mathrm{mM} ; \quad V_{\max , \mathrm{PGI}}=122.5 \mathrm{U} / \mathrm{mg}$, $K_{\mathrm{PGI}, \mathrm{eq}}=0.31 ; \quad K_{\mathrm{PGI}, \mathrm{g} 6 \mathrm{p}}=0.28 \mathrm{mM}, \quad K_{\mathrm{PGI}, \mathrm{f} 6 \mathrm{p}}=0.147 \mathrm{mM}$; $V_{\text {max }, \mathrm{PFK}}=190 \mathrm{U} / \mathrm{mg}, \quad K_{\text {PFK,f6p }}=0.45 \mathrm{mM}, \quad K_{\text {PFK,atp }}=0.018 \mathrm{mM}$, $n=2.2$ ) were taken from Brenda database [15] and the literature [28, 29] and were obtained in conditions similar to the experimental data $(\mathrm{pH}=7.2)$. The experimental data set is from Ishii et al. [27] 


\section{www.ietdl.org}

available kinetic data have several limitations and inconsistencies for constructing dynamic models of metabolism:

1. Large networks are accessible in databases such as MetaCyc [33] and KEGG [30], but the reaction mechanism and reliable rate equations for most reactions remain unknown.

2. Some databases such as EcoCyc [17] and KEGG [30] have inconsistencies on the reversibility of the reactions. For example, on the pathways maps the reaction mechanism for pyruvate oxidase (EC 1.2.2.2) is indicated as reversible in KEGG and as irreversible on EcoCyc. This is because most times reversibility is species dependent as, for the same enzyme, it changes with intracellular conditions. Therefore organism-specific databases usually have more accurate information that can differ from what can be found in multi-organism databases.

3. Kinetic parameters are usually available in repositories like BRENDA [15] and KMedDB [34], which however lack the kinetic expressions describing the associated rate law. This is owing to computational issues, as it is not straightforward to include mathematical formulae in a semiautomatic way in databases. In these cases, parameter values are not really helpful to integrate in the dynamic models. For example, in some works the kinetic law type is available but the respective mathematical equation is not $[35,36]$.

4. In those data sources, for reversible reactions, it is frequent to find only the parameters for the forward and rarely for the reverse reaction or for both [37]. A reason for this is that the reverse reaction is less favourable thermodynamically and therefore difficult to operate assays in those directions.

5. Experimental conditions under which the kinetic parameters had been determined and the methodology of the assay are rarely available. On the other hand, even in the cases where some of this information is available, it refers generally only to temperature and $\mathrm{pH}$. Therefore data standardisation is necessary to reach comparability of enzyme kinetic data and to ensure data quality. A good starting point for the experimentalists to achieve standardisation of the kinetic data in the future is to follow the recent recommendations and guidelines from the STRENDA (standard for reporting enzymology data) commission [38].

6. Equilibrium constants $\left(K_{\text {eq }}\right)$ dependent on temperature and $\mathrm{pH}$ are needed to calculate the steady-state concentrations of the metabolites not experimentally measured, assuming nearequilibrium for the reversible enzyme reactions [39]. However, these important constants are usually not reported in databases and, when available, have often been extracted from conditions different from the physiological ones [40]. This fact can introduce discrepancies between the determined concentration and the real values. Therefore experimentally measured values are required or the recently available database for enzyme catalysed reactions [41] can help to obtain $K_{\text {eq }}$ values as close as possible to the physiological conditions.

7. The maximum velocity $\left(v_{\max }\right)$ depends on the amount of enzyme present and is often measured in test tubes. However, in dynamic modelling we are interested in the in vivo data. Furthermore, the values usually reported in the literature are of specific activities and come as

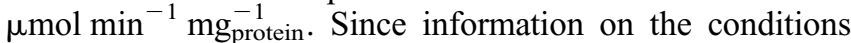
in which the assay was performed is scarce, it is often impossible to convert these units to maximum velocities: if the reported specific activity has been obtained using a

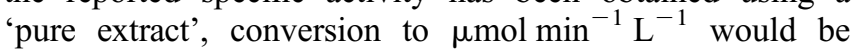
performed by multiplying the specific activities by the values of enzyme concentration (usually in $\mu \mathrm{M}$ ) in the living cell [42] and the molecular weight for the particular enzyme obtained from available sources such as GenoBase [43]; otherwise, if the specific activity has been obtained from a 'crude extract' fraction, the average amount of

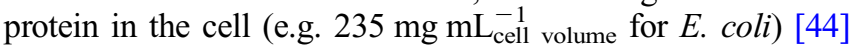
can be used.

8. Sometimes values regarding an inhibition constant are given in literature or databases, but information on the type of inhibition they refer to (competitive, non-competitive, uncompetitive etc.) is not available. Again, this might be related with computational difficulties in representing the kinetic laws.

9. The quality of the determined kinetic parameters, expressed, for example, in confidence intervals and standard errors is seldom available, although this information is important for modelling [45]. This is because of the fact that it is still not a common practice to publish kinetic parameters with confidence intervals.

Although great effort has been carried out by the STRENDA commission in the last few years for the standardisation of the enzyme kinetic data for use in kinetic models, examples can easily be found in the literature where some of these important recommendations are not followed. For example, on the recent publication on in vivo kinetic parameter estimation of E. coli [27] there are missing data, such as on the purity of the enzymes and the accuracy of the parameters (e.g. standard deviation and confidence intervals). More important, however, and crucial

Table 1 Summary of some data sources available online with kinetic information useful for the construction of kinetic models of metabolic pathways

\begin{tabular}{|c|c|c|}
\hline Database & Characteristics & Reference \\
\hline BRENDA & $\begin{array}{l}\text { contains kinetic parameters for several organisms with comments on the experimental conditions } \\
\text { and enzyme kinetics }\end{array}$ & [15] \\
\hline SABIO-RK & $\begin{array}{l}\text { kinetic parameters for various organisms. Associated kinetic equations describing the reaction } \\
\text { rates are also available. Includes experimental conditions from the original paper and reaction } \\
\text { buffer. No information is given about the assay }\end{array}$ & [16] \\
\hline SigPath & collection of parameters and kinetic equations for some organisms, but not for $E$. coli & [31] \\
\hline BioNumbers & offers kinetic and biological data for several organisms & [32] \\
\hline CCDB & $\begin{array}{l}\text { collection of detailed enzymatic data about } E \text {. coli. Mathematical equations and experimental } \\
\text { conditions are not incorporated }\end{array}$ & [14] \\
\hline
\end{tabular}


for the success of dynamic modelling, is the definition of standard assays close to the intracellular conditions. Successful results were obtained in [46], where the authors provide and test a standard 'in vivo-like' assay medium for determining kinetic parameters in yeast systems biology projects. This appears a promising way to overcome some of the obstacles and therefore may serve as a first step for the standardisation of enzymes data.

Furthermore, even when available sources comply with STRENDA requirements, some extensions would make them more useful for modellers, such as the integration of transcriptome, proteome and/or thermodynamics data [47]. These data can be useful when extrapolating the value of the $V_{\max }$ parameter. For example, if we know the gene expression or protein level for two conditions and the $V_{\max }$ for one of them and assuming a proportional change for the two $\left(V_{\max }=k_{\text {cat }}[E]\right)$, the unknown $V_{\max }^{\prime}$ value can be calculated $\left(V_{\max }^{\prime} / V_{\max }=[E]^{\prime} /[E]\right)[48]$.

Also, it is necessary to increase the adoption of the guidelines suggested by the STRENDA commission in the scientific community, including in biochemical journals. To facilitate that, it would be important to develop a repository for the electronic submission of standardised kinetic data and experimental raw data prior to publication, to ensure high accessibility, data quality and data exchange.

We have also observed that the publicly available kinetic databases miss some information important to the modellers that, nevertheless, had been described in the original papers. For example, the most popular database of functional and kinetic enzyme data, BRENDA [15], has limited information about the kinetic parameters for the pyruvate dehydrogenase enzyme (EC 1.2.4.1) (PDH) from E. coli (see Table 2), although the original source contains important details.

Owing to the fact that the collection of kinetic data from the literature is usually carried out manually, some efforts have been recently reported on the application of literature mining approaches for kinetic data extraction [50-52]. The extraction of kinetic parameters using these literature mining algorithms that can be fed with information from standards [53] holds promise in terms of literature coverage and data accuracy. However, this is still a technologically challenging task, most available tools are only semiautomatic and a posterior manual curation by consultation of the literature is required.

Finally, even improving existing resources, available kinetic data are not sufficient for the construction of dynamic models for most organisms and pathways, simply because the involved enzymes have not yet been characterised. There is therefore a need for high-throughput enzyme characterisations to overcome these limitations. One approach in this direction was proposed by Gabdoulline et al. [54] where the authors apply Brownian dynamic simulation, based on protein three-dimensional structures, to estimate the undetermined kinetic parameters. An alternative method, the PIPSA (protein interaction property similarity analysis) methodology [55], was introduced in [56] to estimate parameters based on protein structural information. This approach relates variations in kinetic parameters to difference in the protein structures. Thus, unknown parameters can be estimated for an organism where only a parameter is available for another related organism or obtained under different conditions.

\section{Alternative dynamic modelling approaches}

There are some solutions proposed in the literature to overcome the problems of unavailable kinetic information based on different modelling approaches for large-scale metabolic networks such as statistical frameworks, approximate non-mechanistic kinetic formats or hybrid modelling techniques [57-61]. Such approaches usually help to reduce the number of the total parameters to be estimated and allow estimating parameters from the model structure or other available models.

Dynamic flux balance analysis (dFBA) is a dynamic formulation that allows variation of external metabolite concentrations and assumes an internal pseudo steady state $[62,63]$. It performs time-course simulation by solving a flux balance analysis (FBA) problem [64] at each time step. One advantage of this method is the scalability to genome scale networks. However, this gives no insight into intracellular dynamics and the formulation of the objective function may not be trivial for transient conditions.

In [61], the authors proposed a hybrid modelling approach composed of detailed mechanistic rate equations for the key central carbon metabolism enzymes and approximated kinetics for all the remaining enzymes. A good performance of this hybrid modelling approach was demonstrated for large-scale models. However, one disadvantage is the arbitrary selection of the set of key enzymes. A hybrid modelling approach combining approximated lin-log kinetics and Michaelis-Mentens equations are introduced in [65]. Another hybrid approach was developed by Yugi et al. [60]. The proposed method aims at reducing the number of enzyme kinetic assays necessary to build a dynamic model, by considering a dynamic and a static part. At each time-step the rate laws of the dynamic part are used to calculate its flux distribution. The fluxes at the border between the static and the dynamic part are used in a manner similar to metabolic flux analysis (MFA) [66] to constrain the solution space. As in the previous

Table 2 Kinetic data for PDH enzyme from E. coli as cited in BRENDA and the original reference [49]

\begin{tabular}{lcr}
\hline Parameter(value) & BRENDA (released 02/2008) & Original publication $^{\mathrm{a}}$ \\
\hline$V_{\text {max }}(0.445 \mathrm{U} / \mathrm{mg})$ & wild-type enzyme, & confidence intervals for parameters, description of the \\
& 2,6-dichlorophenolindophenol & spectrophotometric method to measure enzyme activity, \\
$K_{m, \text { pyr }}(0.515 \mathrm{mM})^{\mathrm{b}}$ & assay, substrate pyruvate & reaction medium $\left(50 \mathrm{mM} \mathrm{KH}_{2} \mathrm{PO}_{4}\left(\mathrm{pH}^{\mathrm{b}}=7.0\right), 1 \mathrm{mM} \mathrm{MgCl}_{2}\right.$, \\
$n_{\mathrm{PDH}}(1.38)^{\mathrm{c}}$ & wild-type enzyme & $2.0 \mathrm{C}_{3} \mathrm{H}_{3} \mathrm{NaO}_{3}, 2.5 \mathrm{mM} \mathrm{NAD}^{+}, 0.1-0.2 \mathrm{mM} \mathrm{CoA}, 0.2 \mathrm{mM}$ \\
& no data & ThDP and $2.6 \mathrm{mM}$ dithiothreitol) temperature $=30^{\circ} \mathrm{C}$, \\
& & turnover number $=28.89 \mathrm{~s}^{-1}$ \\
\hline
\end{tabular}

\footnotetext{
anformation not available on BRENDA

${ }^{b}$ Michaelis-Menten constant for the PDH enzyme

${ }^{\mathrm{c}} \mathrm{Hill}$ coefficient
} 
approach, a clear advantage of this method is to take into account the intracellular dynamics. Also, using an MFA instead of an FBA-based approach has the advantage of not requiring an objective function. However, there are some limitations to be considered for accurate simulations, such as the need to obtain elasticity coefficients at boundary reactions between modules and inconsistencies in the static module caused by the inclusion of irreversible reactions. In addition, it limits the scalability of the approach, because, for large-scale networks, the size of the static part would not allow the application of MFA since this part would be significantly larger than the dynamic part.

More recently, mass-action stoichiometric simulation models have been proposed by Jamshidi and Palsson [57, 67] as a way to incorporate kinetic information into stoichiometric reconstructions, using mass-action kinetics for all reactions, and where the kinetic parameters are estimated from equilibrium constants, metabolome and fluxome measurements. A limitation of these models is that mass-action kinetics does not reflect the usual non-linearity of enzymatic reactions. This limitation is overcome by including the mechanistic substrate/enzyme reactions, with the drawback of a dramatic increase in the network size by the inclusion of concentrations of a large number of intermediate reactions.

Alternatively, lin-log models [68] have been used, such as in [69], where the authors have developed a model of the central carbon metabolism in hepatoma cells, and where in vivo time-series data were used for deducing the kinetic parameters and analysing the distribution of metabolic control. The lin-log models constitute an extension of metabolic control analysis, and hence allow analysing the control in large-scale metabolic networks only near the steady state [70]. In both approaches, accounting for intracellular dynamics is a clear advantage.

Smallbone et al. $[59,71]$ proposed a method for combining two modelling approaches (approximated lin-log kinetics and constraint-based modelling), in which the parameters (elasticities) are given by the negative stoichiometric coefficient for the respective metabolites and/or derived from available kinetic models within Biomodels database [72]. The reference steady-state fluxes are estimated by the FBA approach. However, using the negative stoichiometric coefficient values and parameters taken from yeast or other species models are rough estimations and may result in false predictions. In addition, the computational cost of integrating ODE's with hundreds or thousands of equations may become a limiting step.

On the other hand, a method for 'bridging the gap' between the kinetic and structural modelling is introduced in [73]. This approach shows that it is possible to acquire detailed quantitative representation of metabolic networks without explicitly referring to a set of ODE's.

The choice of a modelling approach depends on the available information and the purpose of the kinetic model. Therefore it is impossible to state a priori which approach is more accurate in a general sense. Developing computational approaches for large-scale metabolic networks is hence a major challenge for the near future.

\section{Concluding remarks and future directions}

One key challenge in systems biology is to create computational models for performing in silico experiments. For that purpose, the integration of kinetic information into the models from different research laboratories is required. Therefore there is the need to standardise the experimental procedures and data requirements for modelling purposes.
In this paper, we have described some limitations and inconsistencies detected during the reconstruction of the dynamic metabolic model of $E$. coli based on the literature and public databases, such as missing information on the experimental conditions in which the parameters had been determined. Furthermore, important information that had been reported in the original publications is missing in some kinetic databases. We also propose some suggestions to overcome several of the limitations found.

We hope that in the future, with the standardisation of the in vitro enzyme protocols and the approximation of in vitro conditions to the in vivo ones, it will be possible to integrate available kinetic data into complete large-scale models. We also expect that collaborative projects between modellers and biologists will provide valuable kinetic data and allow the exchange of important information to solve most of these issues.

Finally, while a sufficient amount of standard kinetic data are not available, alternative metabolic modelling approaches have been used that speed up model development, like the hybrid dynamic/static method or approximate kinetics [59, 60].

\section{Acknowledgments}

Rafael S. Costa would like to thank Fundação para a Ciência e Tecnologia for providing the grant SFRH/BD/25506/2005. The authors also acknowledge the MIT-Portugal project 'Bridging Systems and Synthetic Biology for the development of improved microbial cell factories' MIT-Pt/ BS-BB/0082/2008.

\section{References}

1 Ishii, N., Nakahigashi, K., Baba, T., et al.: 'Multiple high-throughput analyses monitor the response of E. coli to perturbations', Science, 2007, 316, (5824), pp. 593-597

2 Zhang, W., Li, F., Nie, L.: 'Integration multiple 'omics' analysis for microbial biology: application and methodologies', Microbiology, 2010, 156, pp. 287-301

3 Kitano, H.: 'Systems biology: a brief overview', Science, 2002, 295, pp. $1662-1664$

4 Monod, J.: 'The growth of bacterial cultures', Annu. Rev. Microbiol., 1949, 3, pp. 371-394

5 Dairaku, K., Izumoto, E., Morikawa, H., Shioya, S., Takamatsu, T.: 'Optimal quality control of Baler's yeast fed-batch culture using population dynamics', Biotechnol. Bioeng., 1982, 24, pp. 2661-2674

6 Blanch, H.W.: 'Microbial growth kinetics', Chem. Eng. Commun., 1981, 8, pp. $181-211$

7 Varner, J.D.: 'Large-scale prediction of phenotype: concept', Biotechnol. Bioeng., 2000, 69, (6), pp. 664-678

8 Chassagnole, C., Noisommit-Rizzi, N., Schmid, J.W., Mauch, K., Reuss, M.: 'Dynamic modeling of the central carbon metabolism of Escherichia coli', Biotechnol. Bioeng., 2002, 79, (1), pp. 53-73

9 Feist, A.M., Herrgard, M.J., Thiele, I., Reed, J.L., Palsson, B.O.: 'Reconstruction of biochemical networks in microorganisms', Nature Rev. Microbiol., 2009, 7, (2), pp. 129-143

10 Fell, D.A.: 'Metabolic control analysis - a survey of its theoretical and experimental development', Biochem. J., 1992, 286, pp. 313-330

11 Blattner, F.R., Plunkett, G.l., Perna, N.T., et al.: 'The complete genome sequence of E. coli $K-12$ ', Science, 1997, 277, (5331), pp. 1453-1462

12 Visser, D.v.Z.A., Heijnen, J.J., Frank, J.: 'Rapid sampling for analysis of in vivo kinetics the BioScope: a system for continuous-pulse experiments', Biotechnol. Bioeng., 2002, 79, (6), pp. 674-681

13 Theobald, U., Mailinger, W., Reuss, M., Rizzi, M.: 'In-vivo analysis of glucose-induced fast changes in yeast adenine-nucleotide pool applying a rapid sampling technique', Anal. Biochem., 1993, 214, (1), pp. 31-37

14 Sundararaj, S., Guo, A., Habibi-Nazhad, B., et al.: 'The CyberCell database (CCDB): a comprehensive, self-updating, relational database to coordinate and facilitate in silico modeling of Escherichia coli', Nucleic Acids Res., 2004, 32, pp. D293-D295

15 Schomburg, I., Chang, A., Schomburg, D.: 'BRENDA, enzyme data and metabolic information', Nucleic Acids Res., 2002, 30, pp. 47-49 
16 Wittig, U., Golebiewski, M., Kania, R., et al.: 'SABIO-RK: integration and curation of reaction kinetic data', Lect. Notes Bioinf., 2006, 4075, pp. $94-103$

17 Karp, P., Riley, M., Saier, M., Paulsen, I.T., Paley, S.M., PellegriniToole, A.: 'The EcoCyc and MetaCyc databases', Nucleic Acids Res., 2000, 28, pp. 56-59

18 Ji, Z.L., Chen, X., Zhen, C.J., et al.: 'KDBI: kinetic data of biomolecular interaction database', Nucleic Acids Res., 2003, 31, pp. 255-257

19 'The database of useful biological numbers', www.bionumbers.org, 2008

20 Grimbs, S., Selbig, J., Bulik, S., Holzhuetter, H.-G., Steuer, R.: 'The stability and robustness of metabolic states: identifying stabilizing sites in metabolic networks', Mol. Syst. Biol., 2007, 3, pp. 146-158

21 Teusink, B., Passarge, J., Reijenga, C.A., et al.: 'Can yeast glycolysis be understood in terms of in vitro kinetics of the constituent enzymes? Testing biochemistry', Eur. J. Biochem., 2000, 267, (17), pp. 5313-5329

22 Rizzi, M., Baltes, M., Theobald, U., Reuss, M.: 'In vivo analysis of metabolic dynamics in Saccharomyces cerevisiae: II. Mathematical model', Biotechnol. Bioeng., 1997, 55, (4), pp. 592-608

23 Goel, G., Chou, I.-C., Voit, E.O.: 'System estimation from metabolic time-series data', Bioinformatics, 2008, 24, (21), pp. 2505-2511

24 Gadkar, K.G., Varner, J., Doyle, F.J.: 'Model identication of signal transduction networks from data using a state regulator problem', IEEE Syst. Biol., 2005, 2, pp. 17-30

25 Wright, B.E., Butler, M., Albe, K.R.: 'Systems analysis of the tricarboxylic acid cycle in Dictyostelium discoideum', J. Biol. Chem., 1992, 267, (5), pp. 3101-3105

26 Bakker, B.M., Michels, P.A.M., Opperdoes, F.R., Westerhoff, H.V.: 'Glycolysis in bloodstream form Trypanosoma brucei can be understood in terms of the kinetics of the glycolytic enzymes', J. Biol. Chem., 1997, 272, (6), pp. 3207-3215

27 Ishii, N., Suga, Y., Hagiya, A., et al.: 'Dynamic simulation of an in vitro multi-enzyme system', FEBS Lett., 2007, 581, (3), pp. 413-420

28 Gao, H., Chen, Y., Leary, J.A.: 'Kinetic measurements of phosphoglucose isomerase and phosphomannose isomerase by direct analysis of phosphorylated aldose-ketose isomers using tandem mass spectrometry', Int. J. Mass Spectrom., 2005, 240, pp. 291-299

29 Ogawa, T., Mori, H., Tomita, M., Yoshino, M.: 'Inhibitory effect of phosphoenolpyruvate on glycolytic enzymes in Escherichia coli', Res. Microbiol., 2007, 158, pp. 159-163

30 Kanehisa, M., Goto, S.: 'KEGG: kyoto encyclopedia of genes and genomes', Nucleic Acids Res., 2000, 28, (1), pp. 27-30

31 Campagne, F., Neves, S., Chang, C., et al.: 'Quantitative information management for the biochemical computation of cellular networks', Science, 2004, 2004, (248), p. pI11

32 www.bionumbers.org, 2008

33 Caspi, R., Foerster, H., Fulcher, C.A., et al.: 'The MetaCyc database of metabolic pathways and enzymes and the BioCyc collection of pathway/ genome databases', Nucleic Acids Res., 2008, 36, pp. D623-D631

$34 \mathrm{http} / / /$ sysbio.molgen.mpg.de/KMedDB, 2010

35 Kreuzberg, K.: 'Interaction of D-fructose and fructose 1-phosphate with yeast phosphofructokinase and its influence on glycolytic oscillations', Biochimica et Biophysica Acta, 1978, 527, (1), pp. 229-238

36 Ruijter, G.J.G., Visser, J.: 'Characterization of Aspergillus niger phosphoglucose isomerase. Use for quantitativa determination of erythrose 4-phosphate', Biochimi, 1999, 81, pp. 267-272

37 Fifif, T., Scopes, R.K.: 'Purification of 3-phosphoglycerate from diverse sources by affinity elution chromatography', Biochem. J., 1978, 175, pp. 311-319

38 STRENDA commission, available at www.strenda.org/documents.html, 2008

39 Schauer, M., Heinrich, R.: 'Quasi-steady-state approximation in the mathematical-modeling of biochemical reaction networks', Math. Biosci., 1983, 65, (2), pp. 155-170

40 Vaseghi, S., Baumeister, A., Rizzi, M., Reuss, M.: 'In vivo dynamics of the pentose phosphate pathway in Saccharomyces cerevisae', Metab. Eng., 1999, 1, pp. 128-140

41 Goldberg, R.N., Tewari, Y.B., Bhat, T.N.: 'Thermodynamics of enzyme-catalyzed reactions - a database for quantitative biochemistry', Bioinformatics, 2004, 20, pp. 2874-2877

42 Lu, P., Vogel, C., Wang, R., Yao, X., Marcotte, E.M.: 'Absolute protein expression profiling estimates the relative contributions of transcriptional and translational regulation', Nature Biotechnol., 2007, 25, (1), pp. 117-124

43 Genome Analysis Project, http://ecoli.naist.jp/GB8/, 2006

44 Albe, K.R., Butler, M.H., Wright, B.E.: 'Cellular concentration of enzymes and their substrates', J. Theor. Biol., 1990, 143, pp. 163-195

45 Wiechert, W., Takors, R.: 'Validation of metaboli models: concepts, tools, and problems', in Kholodenko, B.N., Westerhoff, H.V. (Eds.):
'Metabolic engineering in the post genomic era' (Horizon Bioscience, 2004), pp. 277-318

46 van Eunen, K., Bouwman, J., Daran-Lapujade, P., et al.: 'Measuring enzyme activities under standardized in vivo-like conditions for systems biology', FEBS J., 2010, 277, pp. 749-760

47 Alberty, R.A.: 'Biochemical thermodinamics: applications of mathematica', Methods Biochem. Anal., 2006, 48, pp. 1-458

48 Oh, M.K., Rohlin, L., Kao, K.C., Liao, J.C.: 'Global expression profiling of acetate-grown Escherichia coli', J. Biol. Chem., 2002, 277, (15), pp. $13175-13183$

49 Nemeria, N., Yan, Y., Zhang, Z., et al.: 'Inhibition of the Escherichia coli pyruvate dehydrogenase complex E1 subunit and its tyrosine 177 variants by thiamin 2-thiazolone and thiamin 2-thiothiazolone diphosphates. Evidence for reversible tight-binding inhibition', J. Biol. Chem., 2001, 276, pp. 45969-45978

50 Hakenberg, J., Schmeier, S., Kowald, A., Klipp, E., Leser, U.: 'Finding kinetic parameters using text mining', Omics, J. Integr. Biol., 2004, 8 , (2), pp. 131-152

51 Spasic, I., Simeonidis, E., Messiha, H.L., Paton, N.W., Kell, D.B.: 'KiPar, a tool for systematic information retrieval regarding parameters for kinetic modelling of yeast metabolic pathways', Bioinformatics, 2009, 25, (11), pp. 1404-1411

52 Heinen, S., Thielen, B., Schomburg, D.: 'KID - an algorithm for fast and efficient text mining used to automatically generate a database containing kinetic information of enzymes', BMC Bioinf., 2010, 11, pp. $375-388$

53 Le Novère, N.: 'Model storage, exchange and integration', $B M C$ Neurosci., 2006, 7, (Suppl 1), p. S11

54 Gabdouline, R.R., Kummer, U., Olsen, L.F., Wade, R.C.: 'Concerted simulation reveal how peroxidase compind III formatio results in cellular oscillations', Biophys. J., 2003, 85, (3), pp. 1421-1428

55 Wade, R.C., Gabdoulline, R.R., De Rienzo, F.: 'Protein interaction property similarity analysis', Int. J. Quant. Chem., 2001, 83, pp. $122-127$

56 Gabdoulline, R.R., Stein, M., Wade, R.C.: 'qPIPSA: relating enzymatic kinetic parameters and interaction fields', BMC Bioinf., 2007, 8, pp. $373-389$

57 Jamshidi, N., Palsson, B.O.: 'Mass action stoichiometric simulation models: incorporating kinetics and regulation into stoichiometric models', Biophysical Journal, 2010, 98, pp. 175-185

58 Resendis-Antonio, O.: 'Filling kinetics gaps: dynamic modeling of metabolism where detailed kinetic information is lacking', PLoS ONE, 2009, 4, (3), pp. 1-11

59 Smallbone, K., Simeonidis, E., Broomhead, D.S., Kell, D.B.: 'Something from nothing - bridging the gap between constraint-based and kinetic modelling', FEBS J., 2007, 274, (21), pp. 5576-5585

60 Yugi, K., Nakayama, Y., Kinoshita, A., Tomita, M.: 'Hybrid dynamic/ static method for large-scale simulation of metabolism', Theor. Biol. Med. Model., 2005, 2, pp. 42-53

61 Bulik, S., Grimbs, S., Huthmacher, C., Selbig, J., Holzhuetter, H.G. 'Kinetic hybrid models composed of mechanistic and simplified enzymatic rate laws - a promising method for speeding up the kinetic modelling of complex metabolic networks', FEBS J., 2009, 276, pp. $410-424$

62 Mahadevan, R., Edwards, J.S., Doyle, F.J.: 'Dynamic flux balance analysis of diauxic growth in Escherichia coli', Biophys. J., 2002, 83, (3), pp. $1331-1340$

63 Oddone, G.M., Mills, D.A., Block, D.E.: 'A dynamic, genome-scale flux model of Lactococcus lactis to increase specific recombinant protein expression', Metab. Eng., 2009, 11, (6), pp. 367-381

64 Raman, K., Chandra, N.: 'Flux balance analysis of biological systems: applications and challanges', Brief. Bioinf., 2009, 10, (4), pp. 435-449

65 Costa, R.S., Machado, D., Rocha, I., Ferreira, E.C.: 'Hybrid dynamic modeling of Escherichia coli central metabolic network combining Michaelis-Menten and approximate kinetic equations', Biosystems, 2010, 100, (2), pp. 150-157

66 Lee, K., Berthiaume, F., Stephanopoulos, G.N., Yarmush, M.L.: 'Metabolic flux analysis: a powerful tool for monitoring tissue function', Tissue Eng., 1999, 5, (4), pp. 347-368

67 Jamshidi, N., Palsson, B.O.: 'Formulating genome-scale kinetic models in the post-genome era', Mol. Syst. Biol., 2008, 4, pp. 171-180

68 Visser, D., Heijnen, J.J.: 'Dynamic simulation and metabolic re-design of a branched pathway using linlog kinetics', Metab. Eng., 2003, 5, (3), pp. $164-176$

69 Maier, K., Hofmann, U., Reuss, M., Mauch, K.: 'Dynamics and control of the central carbon metabolism in hepatoma cells', BMC Syst. Biol., 2010, 4, pp. 54-82

70 Heinrich, R., Rapoport, T.A.: 'Linear steady-state treatment of enzymatic chains - general properties, control and effector strength', Eur. J. Biochem., 1974, 42, (1), pp. 89-95 


\section{www.ietdl.org}

71 Smallbone, K., Simeonidis, E., Swainston, N., Mendes, P.: 'Towards a genome-scale kinetic model of cellular metabolism', BMC Syst. Biol., 2010, 4, (6), pp. 1-9

72 Le Novere, N., Bornstein, B., Broicher, A., et al.: 'BioModels database: a free, centralized database of curated, published, quantitative kinetic models of biochemical and cellular systems', Nucleic Acids Res., 2006, 34, pp. D689-D691

73 Steuer, R., Gross, T., Selbig, J., Blasius, B.: 'Structural kinetic modeling of metabolic networks', Proc. Natl. Acad. Sci. (USA), 2006, 103, pp. $11868-11873$ 